\title{
Hge-Related Changes in Doxorubicin-Induced Oxidative Damage: Protective and Pretreatment Effects of Short-term Herobic Exercise
}

\author{
Seyyede Zolaikha Hashemi \\ Chashmi(MSc) \\ Department of Sport Physiology, \\ Faculty of Physical Education and \\ Sport Sciences, University of \\ Mazandaran, Babolsar, Iran
}

Valiollah Dabidi Roshan(PhD) Department of Sport Physiology, Faculty of Physical Education and Sport Sciences, University of Mazandaran, Babolsar, Iran

\section{Sohail Azizi (MD)}

Department of Laboratory Medicine, Faculty of Allied Medical Sciences, Mazandaran University of Medical Sciences, Sari, Iran

Corresponding author: Valiollah Dabidi Roshan

Email: v.dabidi@umz.ac.ir

Tel: +989113151509

Address: University of Mazandaran, Babolsar, Iran

Received : 20 Feb 2016

Revised: 25 Mar 2016

Accepted: 10 Mar 2016
ABSTRACT

Background and 0bjective: Doxorubicin (D0X) is an effective anticancer drug. It has been shown that a short-term exercise performed prior to DOX-treatment has no effect on cardiotoxicity in young rats. In the present study, old and young rats were evaluated to determine the protective effects of pre-treatment with short-term exercise on D0X-induced oxidative damage in cardiac tissue.

Methods: Forty-eight male Wistar rats were randomly divided into two groups of young and old, and later divided into three sub-groups of young+D0X, young+training+DOX, young+training+salin, old+D0X, old+training+D0X and old+training+salin. The training protocol included treadmill running for $25-39 \mathrm{~min} /$ day at $15-17 \mathrm{~m} / \mathrm{min}, 5$ days/week for three weeks. All treatments were carried out $24 \mathrm{~h}$ after the last exercise bout. The rats were sacrificed $48 \mathrm{~h}$

after DOX administration.

Results: Although D0X injection significantly affected the cardiac tissue of old rats compared to young rats, pretreatment with endurance training in D0X-treated rats caused an increase in Heat shock protein (3.02\% vs. 23.36) and superoxide dismutase (30.12\% vs. 31.12 ), and a decrease in malondialdehyde (10.92\% vs. 19.60$)$ in both old and young rats.

Conclusion: Although DOX-induced production of free radicals and cardiotoxicity in aged rats is more than that in young rats, the short-term aerobic exercise reduced the damaging effects of free radicals in the old rats more than in young ones. The concentration of antioxidant enzymes also increases with exercise in the old rats compared to young rats.

Keywords: Cardiotoxicity, Doxorubicin, 0xidative Stress, Aerobic Exercise. 


\section{INTRODUCTION}

Doxorubicin (DOX) is one of the most effective chemotherapeutic factors used in the treatment of various types of cancer $(1,2)$. However, the clinical use of DOX is often limited because of its serious toxic side effects on different organs such as the heart, liver, etc.(3). Most studies confirm that oxidative stress plays an important role in the pathogenesis of DOX-induced toxicity $(4,5)$.

Several mechanisms have been proposed to be attributed for the DOX-induced cardiotoxicity including free radicals, lipid peroxidation, mitochondrial damage and cellular toxicity $(6,7)$. The overproduction of free radicals and oxidative stress in violation of anti-oxidants plays a main role in the development of DOXinduced tissue damage $(8,9)$. Malondialdehyde (MDA) is a marker of lipid peroxidation. Evidence suggests that the increased oxidative stress due to increased free radical production and decreased myocardial endogenous antioxidants play important roles in the pathogenesis of heart failure.

Aging is caused by accumulation of molecular damage in DNA $(10,11)$. Oxidative stress increases with age due to macromolecular damage and/or an imbalance between reactive oxygen/nitrogen species production and antioxidant defense $(12,13)$. In addition, the incidence of cancer is high among the elderly $(14,15)$, demonstrating the role of oxidative stress in aging-associated diseases $(16,17)$. Therefore, it is necessary to find the strategies that reduce the adverse effects of oxidative stress and DOX treatment in this age group, and cancer-treated elderly in particular. In contrast, several studies have reported that an organism is generally protected from free radicals damage via its antioxidant defense system, which is modulated by administration of DOX (18). The cellular antioxidant defense system operates mainly via antioxidant enzymes, such as superoxide dismutase (SOD), and independent from non-enzymatic antioxidants such as heat protein shock (HSP) (19). Induction of $\mathrm{HSP}_{70}$ is known to occur in myocardial tissue following exercise training, which is associated with the exercise-induced preservation of cardiac function during states of oxidative stress $(19,20)$. Primary functions of HSP include protection against apoptosis and oxidative damage $(17,21)$. According to previous studies, the main function of antioxidants is the detoxification of reactive species and toxic oxygen metabolites generated by the endogenous and exogenous metabolism pathways (22).

Heart is one of the most affected organs in DOX therapy (23). Therefore, it is necessary to seek for complementary and alternative strategies against DOX-induced cardiotoxicity. Although, there is evidence that acute exercise results in oxidative stress and cardiac damage (24), it seems that regular endurance training could be helpful for prevention and/or treatment of several diseases. Moreover, while most recent studies have focused on the beneficial effect of endurance exercise in DOX-induced cardiotoxicity among young adults, most elderly cancer patients have been relatively neglected. Thus, the increased quality of life in this group of people is essential (25). A new insight could consist of recognizing regular training as a nonpharmaceutical therapeutic strategy for treatment with DOX. There is a hypothesis that DOX-induced cardiotoxicity may be related to free radical formation and oxidative stress, and enhanced antioxidant/oxidation ratio after regular endurance exercise may protect against DOX-induced toxicity in cardiac tissue. Therefore, this study investigated the protective effects of pretreatment with short-term aerobic exercise on oxidative damage, $\mathrm{HSP}_{70}$, SOD and MDA of young and old rats acutely exposed to DOX.

\section{MATERIAL AND METHODS}

The male Wistar rats were divided into two groups based on age ( 3 and 32 months). The animals were then randomly separated into subgroups of control+DOX (20 mg/kg, $\mathrm{n}=8)$, training+DOX $(\mathrm{T}+\mathrm{DOX} 20 \mathrm{mg} / \mathrm{kgn}=8)$, and 
training+saline $(\mathrm{T}+\mathrm{S})$. the Subjects were habituated to treadmill running for 5 days (10 min exercise/day at $10 \mathrm{~m} / \mathrm{min}, 0 \%$ grade). The exercise training program was performed on treadmill with zero slopes for 25-39 $\mathrm{min} / \mathrm{session}$ at $15-17 \mathrm{~m} / \mathrm{min}, 5$ days/week for three weeks. The mentioned exercise training was performed according to the protocol described by Ashrafi et al. (Table 1) (3). DOX was obtained from EBEWE Pharma Ges.m.b.H.Nfg, KG (A-4866 unterach, Austria) as a vial of ph.Eur. DOX was dissolved in $0.9 \%$ saline to prepare a concentration of $20 \mathrm{mg} / \mathrm{kg}$, which is the clinical dose pharmacologically scaled for use in rats (16).

The rats in all groups were anesthetized with ketamine $(30-50 \mathrm{mg} / \mathrm{kg}$ of body weight, ip) and xylazine (3-5 $\mathrm{mg} / \mathrm{kg}$ of body weight, ip) after 10-12 hours of overnight fasting. The subjects were placed supine, the abdominal cavity was opened to expose left ventricle, and 2-ml blood samples were collected in tubes. Then, hearts were quickly excised, rinsed, carefully dried, weighed and placed into Petri dishes containing cold isolation medium $(0.1 \mathrm{~mol} / \mathrm{L} \mathrm{K} 2 \mathrm{HPO} 4$, $0.15 \mathrm{~mol} / \mathrm{L} \mathrm{NaCl}$, at $\mathrm{pH} 7.4$ ) to remove the blood. They were frozen immediately in liquid nitrogen and stored at $-80{ }^{\circ} \mathrm{C}(22,23)$. The heart samples were homogenized in a homogenization buffer $(0.05 \mathrm{M}$ Tris, $0.03 \mathrm{M}$ Lserine, and $0.06 \mathrm{M}$ boric acid, $\mathrm{pH} 7.6 ; 100 \mathrm{mg}$ of tissue/ml of buffer). $\mathrm{HSP}_{70}$ was measured using a commercially available enzyme linked immunosorbent assay (ELISA) kit (Zellbio Co., Germany). First, $100 \mu$ l of standard, blank and sample were added to each well. The liquid was removed from each well and $100 \mu \mathrm{l}$ of biotinantibody working solution were added to each well. Each well was aspirated and washed three times. Then, $100 \mu \mathrm{l}$ of HRP-avidin working solution was added to each well. The aspiration and washing process were repeated five times in the fourth step. Moreover, $90 \mu \mathrm{l}$ of 3,3',5,5'Tetramethylbenzidine substrate was added to each well. Then, $50 \mu$ l of stop solution was added to each well, while the first four wells containing the highest concentration of standards showed an obvious blue color.
Finally, the optical density of each well was determined within 30 minutes at $450 \mathrm{~nm}$ using a microplate reader. Later, SOD activity and MDA content of the supernatants were evaluated using commercial assay kits (Zellbio Co., Germany) according to the method described by Chularojmontri et al. $(24,25)$. One-way analysis of variance (Stat Soft, Inc., Tulsa, OK) was used to identify significant differences among the groups. A post-hoc test (Scheffe's test) was performed to determine differences in levels of different biomarkers among the groups. The P-value less than 0.05 was considered statistically significant.

\section{RESULTS}

Mean levels of $\mathrm{HSP}_{70}$, SOD and MDA in tested rats that have been acutely exposed to DOX $(20 \mathrm{mg} / \mathrm{kg})$ are shown in Table 2 . Administration of $20 \mathrm{mg} / \mathrm{kg}$ DOX in the old rats caused insignificant increases in MDA $(5.55 \%)$ and $\mathrm{HSP}_{70}(17.57 \%)$ levels, while causing an insignificant decrease in SOD levels $(3.86 \%)$ when compared to the young rats. Moreover, three weeks of aerobic exercise in the old group caused a significant increase $(16.89 \%)$ in the MDA, an insignificant decrease $(1.81 \%)$ in the $\mathrm{HSP}_{70}$ and an insignificant decrease $(4.20 \%)$ in SOD level compared to the young group.

After three weeks of aerobic exercise, $\mathrm{HSP}_{70}$ level increased in the young and old groups $(23.36 \%$ vs. $3.02 \%)$. There was a significant increase in SOD level (31.12\% vs. $30.60 \%$ ) and a significant decrease in MDA level $(19.60 \%$ vs. $10.64 \%$ ) in comparison with the control+DOX group.

Moreover, three weeks of pretreatment with aerobic exercise caused a significant and an insignificant increase in $\mathrm{HSP}_{70}$ level of young rats and old rats in the training+salin group, respectively. The SOD level increased significantly in young rats and insignificantly in old rats. MDA levels of young and old rats in the training+salin group decreased significantly compared to training+DOX and control+DOX groups. 
Table1- Exercise training protocol used in the present study

\begin{tabular}{|c|c|c|c|c|}
\hline \multirow{2}{*}{\multicolumn{2}{|c|}{ Training sessions and variables }} & \multicolumn{3}{|c|}{ Weeks of training } \\
\hline & & 1 & 2 & 3 \\
\hline & speed $*$ & 15 & 16 & 17 \\
\hline 1 & duration \# & 25 & 30 & 35 \\
\hline & speed & 15 & 16 & 17 \\
\hline 2 & duration & 26 & 31 & 36 \\
\hline & speed & 15 & 16 & 17 \\
\hline 3 & duration & 27 & 32 & 37 \\
\hline & speed & 15 & 16 & 17 \\
\hline 4 & duration & 28 & 33 & 38 \\
\hline & speed & 15 & 16 & 17 \\
\hline 5 & duration & 29 & 34 & 39 \\
\hline
\end{tabular}

*Meter/min; \# min/session

Table2- Effect of pretreatment with aerobic exercise and DOX treatment on oxidative stress-related biomarkers in the cardiac tissue of subjects in different groups.

\begin{tabular}{|c|c|c|c|c|c|c|}
\hline Markers & & & & & & \\
\hline $\begin{array}{r}\text { Groups } \\
\end{array}$ & $\mathrm{Y}+\mathrm{DOX}$ & $\mathrm{Y}+\mathrm{T}+\mathrm{DOX}$ & $\mathbf{Y}+\mathbf{T}+\mathbf{S}$ & E +DOX & E+T+DOX & $\mathbf{E}+\mathbf{T}+\mathbf{S}$ \\
\hline $\begin{array}{l}\mathrm{HSP}_{70}(\mathbf{n g} / \mathrm{mg} \\
\text { protein) }\end{array}$ & $21.4 \pm 2.44$ & $26.4 \pm 1.98$ & $30.2 \pm 2.86$ & $25.16 \pm 3.6$ & $25.92 \pm 4.74$ & $27.99 \pm 5.55$ \\
\hline $\operatorname{SOD}(\mu /$ mg protein $)$ & $75.31 \pm 6$ & $98.75 \pm 2.75$ & $112.23 \pm 3.06$ & $72.43 \pm 4.84$ & $94.6 \pm 6.83$ & $102.87 \pm 6.46$ \\
\hline $\begin{array}{l}\text { MDA(nm/mg } \\
\text { protein }\end{array}$ & $58.66 \pm 7.97$ & $47.16 \pm 10.15$ & $22.35 \pm 4.25$ & $61.89 \pm 4.93$ & $\mathbf{5 5 . 1 3} \pm 6.97$ & $27.61 \pm 3.95$ \\
\hline
\end{tabular}

Data are presented as the Mean \pm SD for 8 rats; Y+ DOX (young +20 mg.kg- $\left.{ }^{-1} D O X\right), Y+T+$ DOX (young+training +20 mg.kg ${ }^{-1}$ DOX), Y $+\mathrm{T}+\mathrm{S}$ (young+training+salin), E+DOX (old + 20 mg.kg- ${ }^{-1}$ DOX), E+T+DOX (old + training + 20 mg.kg ${ }^{-1}$ DOX), E+T+S (old +training+salin).

\section{DISCUSSION}

Previous studies have focused on the role of physical activity as a non-pharmaceutical strategy for treatment of cancer. To the best of our knowledge, we are the first to investigate the effect of pretreatment with short-term endurance training before DOX administration on markers related to cardioprotection and cardiac stress in old and young rats. The results indicated a potential relationship between aged-associated oxidative stress and DOX-induced cardiac stress. In addition, the data suggested that pretreatment with regular short-term aerobic exercise could prevent increased oxidative stress caused by DOX administration by improving antioxidants' activity. These findings helped in understanding how regular physical exercise, particularly running on a treadmill, could contribute to augmentation of cardiac muscle against DOX-induced cardiac stress (26). In this regard, Kavazis, Chicco and Ascensao reported that intraperitoneal injection of $20 \mathrm{mg} / \mathrm{kg}$ of DOX to rats significantly increases $\mathrm{HSP}_{70}$, cardiac malfunction, and coronary blood flow decreases SOD expression and increases lipid peroxidation (MDA) (17, $22,23)$.

The use of DOX is limited for chemotherapy due to its toxicity $(26,27)$. It disrupts the oxidant-antioxidant systems, resulting in tissue damage caused by lipid peroxidation and protein oxidation (28). The present study revealed severe biochemical changes as well as an oxidative damage in cardiac tissue after treatment with $20 \mathrm{mg} \cdot \mathrm{kg}^{-1}$ of DOX. However, the exact mechanism of DOX-induced cardiac stress remained unclear. Nevertheless, a large body of evidence indicates the formation of oxygen free radicals, which can damage cells through lipid peroxidation $(7,29)$. Free radicals are continuously produced in vivo, and there are a number of protective antioxidant enzymes (SOD, catalase, etc.) for dealing with toxic substances. The delicate balance between production and catabolism of oxidants is 
critical for maintaining biological functions (6, 30). According to the results of the present study, regular endurance training decreased the level of MDA and cardiac disturbances induced by administration of acute single dose of DOX. Considering the changes observed in SOD (partially) and $\mathrm{HSP}_{70}$ of rats treated with DOX in response to training, these markers might be taken into account as essential cellular defenses against free radical-based DOX-induced cardiotoxicity, providing enhanced tolerance to trained myocardium at least in the first $48 \mathrm{~h}$ after the end of training period. Moreover, the three weeks of aerobic training and DOX administration (20mg.kg-1) significantly increased $\mathrm{HSP}_{70}$ and $\mathrm{SOD}$, while causing a significant decrease in MDA compared to the control+DOX group (20mg.kg-1). However, the findings demonstrated that the exercise could protect the heart against DOX-induced damage $(3,31)$. These findings are consistent with the findings of our previous study that showed the cardioprotective role of endurance training in DOX-treated rats (22). Since DOXinduced cardiotoxicity has a marked oxidative etiology (32), and chronic exercise improves cardiac capacity of antioxidant systems to counteract with adverse ROS effects (33), it can be suggested that the exercise traininginduced protection against DOX could be partially attributed to improvement in the cardiac antioxidant systems. There were three possible pathways to explain the protective effects of regular endurance exercise against DOX-induced cardiac stress. Aging is a multifactorial process resulting in damage of molecules, cells, and tissues. It has been demonstrated that the expression and activity of antioxidant systems (SOD, HSP) are modified in aging, with reducing cell ability to counteract the oxidant molecules, and consequent weak resistance to ROS accumulation. Physical activity is one of the best methods in treatment of age-related chronic-degenerative diseases and in prevention of changes associated with aging. It also increases the expression and activity of antioxidant enzymes, with consequent reduction of ROS. Positive effects of physical exercise on antioxidant activity could be ascribable to a greater expression and activity of SOD enzymes and $\operatorname{HSP}_{70}(17,25)$. Currently, the principal mechanism of DOXinduced cardiotoxicity is believed to be increased oxidant production by the mitochondria $(22,23)$, which is a main site of ROS production. Exercise seems to increase the oxygen consumption rate by 10-20 fold, and might have released the above factors and thereby induced heart-SOD activity (3). The phenomenon usually referred as crosstolerance has been demonstrated by several studies in which endurance training seems to up-regulate cardiac antioxidant systems and mitochondrial function, to reduce the formation of lipid peroxidation by-products and induce antioxidant defenses such as SOD and HSP after certain stress stimuli $(22,23)$. Our results also support the concept that oxidant/antioxidant imbalance could be the primary event in DOX-induced cardiotoxicity (22). The study of Rinaldi et al. showed that a physical training program increased the level of SOD and $\mathrm{HSP}_{70}$ in trained old rats compared to sedentary old and young rats. Physical activity is able to reduce the generation of oxidants during ischemiareperfusion injury and plays a calciumprotective role via activation of the ROS scavenger enzyme (MnSOD). This improved oxidative status consequent to a correct physical activity program is partially responsible for some benefits such as decreased arterial stiffness, improved endothelial function, metabolic and clotting setting, and reduced body weight $(31,34)$. An interesting finding that may provide further insight into the effects of DOX on the myocardium was a slight decrease in $\mathrm{HSP}_{70}$ protein content in the heart of DOX-treated rats (36). Induction of $\mathrm{HSP}_{70}$ is known to occur in myocardial tissue following exercise training and is associated with the exerciseinduced preservation of cardiac function 
During oxidative stress regardless of its cardioprotective role. Over-expression of HSP could be interpreted as an acute sign of cellular stress (25). Hence, given the vast protective properties of $\mathrm{HSP}_{70}$, we hypothesized that exercise-induced increases in myocardial $\mathrm{HSP}_{70}$ level played an important role in exercise-induced cardioprotection against DOX-mediated cardiac injury $(11,35)$. Therefore, regular endurance training caused a significant increase in the $\mathrm{HSP}_{70}$ and SOD activity and a decrease in MDA of cardiac tissue of subjects in the training+DOX groups.

\section{CONCLUSION}

The present study suggests that DOX treatment be associated with oxidant/antioxidant imbalance in cardiac

\section{REFERENCES}

1. Lien C, Brock T, Jensen S, Hayward H. Short-term exercise training attenuates acute doxorubicin cardiotoxicity. J Physiol Biochem. 2015; 71(4): 669-78. doi: 10.1007/s13105-015-0432-x.

2. Naylor-Dirks JA, Kouzi S A, Yang S, Tran NT, Bero $\mathrm{JD}$, Mabolo R, et al. Can short-term fasting protect against doxorubicininduced cardiotoxicity? World Journal of Biological Chemistry. 2014; 5(3): 269-74. doi: 10.4331/wjbc.v5.i3.269.

3. Ahrafi J, Roshan VD, Mahjoub S. Cardioprotective effects of aerobic regular exercise against doxorubicininduced oxidative stress in rat. African Journal of Pharmacy and Pharmacology. 2012; 6(31): 2380-8.

4. Rašković A, Stilinović N, Kolarović J, Vasović V, Vukmirović S, Mikov M. The Protective Effects of Silymarin against Doxorubicin-Induced Cardiotoxicity and Hepatotoxicity in Rats. Molecules 2011; 16(10): 8601-13. doi: 10.3390/molecules16108601.

5. Nazmi AS, Ahmad SJ, Pillai KK, Akhtar M, Ahmad A, Najmi AK. Protective effects of Bombyx mori, quercetin and benazepril against doxorubicin induced cardiotoxicity and nephrotoxicity. Journal of Saudi Chemical Society. 2013:1-5,6.

6. Zolfagharzadeh F, Roshan VD. Pretreatment Hepatoprotective Effect of Regular Aerobic Training against Hepatic Toxicity Induced by Doxorubicin in Rats. . Asian Pacific J Cancer Prev. 2013;14:2931-6.

7. Marques-Aleixo I, Santos-Alves E, Mariani D, RizoRoca D, Padrão AI, Rocha-Rodrigues S, et al. Physical exercise prior and during treatment reduces sub-chronic doxorubicin-induced mitochondrial toxicity and oxidative stress. Mitochondrion. 2015; 20: 22-33. doi: 10.1016/j.mito.2014.10.008.

8. P Simončíková, A Ravingerová, Barancík M. The effect of chronic doxorubicin treatment on mitogenactivated protein kinases and heat stress proteins in rat hearts. Physiol Res. 2008; 57: s97-s102. tissue. The present study provides new insights into the biochemical mechanisms through which pre-treatment with endurance exercise training exerts its potential antioxidant properties, and protects cardiac tissue against the DOX-induced toxicity.

\section{ACKNOWLEDGMENTS}

This article is the result of a $\mathrm{PhD}$ thesis, approved by the Mazandaran University, Babolsar. We would like to thank all those who participated and worked in this study.

\section{CONFLICT OF INTEREST}

The authors declare that they have no conflict of interests.

9. orrenius S, nicotera $\mathrm{p}$, zhivotovsky B. Cell death mechanisms and their implications in toxicology. Toxicological sciences. 2011;119(1): 3-19. doi: $10.1093 /$ toxsci/kfq268.

10. Reuter S, Gupta SC, Chaturvedi MM, Aggarwal BB. Oxidative stress, inflammation, and cancer: How are they linked? Free Radic Biol Med. 2010; 49(11): 160316.

11. Minelli A, Bellezza I, Conte C, Culig Z. Oxidative stress-related aging: A role for prostate cancer? Biochim Biophys Acta. 2009; 1795(2): 83-91. doi: 10.1016/j.bbcan.2008.11.001

12. Coskun PE, Busciglio J. Oxidative Stress andMitochondrial Dysfunction in Down's Syndrome: Relevance to Aging and Dementia. Hindawi Publishing Corporation. 2012; (2012): Article ID 383170.

13. Lieu CA, Chinta SJ, Rane A, Andersen JK. AgeRelated Behavioral Phenotype of an Astrocytic Monoamine Oxidase-B Transgenic Mouse Model of Parkinson's Disease. PLOS ONE. 2013; 8(1): 1-7.

14. Greenwald P. Clinical Trials in Cancer Prevention: Current Results and Perspectives for the Future. American Society for Nutritional Sciences. 2004; 134(12): 3507S-12S

15. Locke M, Noble EG, Tanguay RM, Feild MR, Ianuzzo SE, Ianuzzo CD. Activation of heat-shock transcription factor in rat heart after heat shock and exercise. American Journal of Physiology - Cell Physiology. 1995; 268(6): C1387-C94.

16. Powers S, Locke AM, Demirel HA. Exercise, heat shock proteins, and myocardial protection from $I-R$ injury. Med Sci Sports Exerc. 2001; 33: 386-92.

17. Kavazis A, Smuder A, Min K, Tumer N, Powers S. Short-term exercise training protects against doxorubicin-induced cardiac mitochondrial damage independent of HSP72. Am J Physiol Heart CircPhysiol. 2010; 299(5): 515-24. 
18. Ascensao A, Oliveira P, Magalhaes J. Exercise as a beneficial adjunct therapy during Doxorubicin treatment--role of mitochondria in cardioprotection. Int $\mathrm{J}$ Cardiol. 2012; 156(1): 4-10.

19. Ascensao A, Lumin-Oliveira J, Machado NG, Ferreira RM, Goncaalves IO, Moreira AC, et al. Acute exercise protects against calcium-induced cardiac mitochondrial permeability transition pore opening in doxorubicin-treated rats. Clinical Science 2011; 120(1): $37-49$.

20. Carvalho FS, Burgeiro A, Garcia R, Moreno AJ, Carvalho RA, Oliveira PJ. Doxorubicin-Induced Cardiotoxicity. Medicinal Research Reviews. 2014; 34: 106-35.

21. Shirinbayan V, Roshan VD. Pretreatment Effect of Running Exercise on HSP70 and DOXInduced Cardiotoxicity. Asian Pacific J Cancer Prev. 2012; 13(11): 5849-55.

22. Ascensao A, Magalhaes J, Soares J, Ferrirra R, Neuparth M, Marques F, et al. Endurance training attenuates doxorubicin-induced cardiac oxidative damage in mice. Int J Cardiol. 2005; 100(3): 451-60.

23. Chicco A, Hydock D, Schneider C, Hayward R. Lowintensity exercise training during doxorubicin treatment protects against cardiotoxicity. J Appl Physiol. 2006; 100(2): 519-27.

24. Chularojmontri L, Wattanapitayakul SK, Herunsalee A, Charuchongkolwongse S, Ninumsakul S, Srichairat S. Antioxidative and Cardioprotective Effects of Phyllanthus urinaria L. on Doxorubicin-Induced Cardiotoxicity. Biol Pharm. 2005; 28(7): 1165-71.

25. Shirinbayan V, Roshan VD, Mahjoub S. The Therapeutic Effect of Endurance Training on Adriamycin-induced Cardiac Stress in Rats. Iranian Journal of Health and Physical Activity. 2013; 4(2): 817.

26. Soliman HAE, Ahmed RR, Gomaa HA, Ali AT. Assessment of the chemo-preventive effects of various plant constituents against doxorubicin-induced toxicity in rats. Journal of American Science. 2014; 10(9): 153-64.
27. Kirkham AA, Davis MK. Exercise Prevention of Cardiovascular Disease in Breast Cancer Survivors. Journal of Oncology. 2015; (2015): Article ID 917606:114.

28. Sturgeon K, Schadler K, Muthukumaran G, Ding D, Bajulaiye A, Thomas NJ, et al. Concomitant low-dose doxorubicin treatment and exercise. Am J Physiol Regul Integr Comp Physiol 2014; 307(6): R685-R92. DOI: 10.1152/ajpregu.00082.2014.

29. Scott JM, Khakoo A, Mackey JR, Haykowsky MJ, Douglas PS, Jones LW. Modulation of AnthracyclineInduced Cardiotoxicity by Aerobic Exercise in Breast Cancer: Current Evidence and Underlying Mechanisms. Circulation. 2011; 124(5): 642-50.

30. Powers SK, Malcolm JJ. Exercise-Induced Oxidative Stress: Cellular Mechanisms and Impact on Muscle Force Production. Physiol Rev. 2008;88(4):1243-76. doi: 10.1152/physrev.00031.2007.

31. Rinaldi B, Corbi G, Boccuti S, Filippelli W, Rengo $\mathrm{G}$, Leosco D, et al. Exercise training affects age-induced changes in SOD and heat shock protein expression in rat heart. Exp Gerontol. 2006; 41(8): 764-70.

32. Wallace K. Doxorubicin-induced cardiac mitochondrionopathy. Pharmacol Toxicol. 2003; 93(3): 105-15.

33. Corbi G, Conti V, Russomanno G, Rengo G, Vitulli $\mathrm{P}$, Ciccarelli AL. Is Physical Activity Able toModify Oxidative Damage in Cardiovascular Aging? Oxidative Medicine and Cellular Longevity. 2012; 2012: 728547. doi: 10.1155/2012/728547.

34. Naka K, Vezyraki P, Kalaitzakis A, Zerikiotis S, Michalis L, Angelidis C. Hsp70 regulates the doxorubicin-mediated heart failure in Hsp70-transgenic mice. Cell Stress and Chaperones. 2014; 19(6): 853-64. doi: 10.1007/s12192-014-0509-4.

35. Powers S, Kavazis A, McClung J. Oxidative stress and disuse muscle atrophy. J Appl Physiol. 2007; 102(6): 2389-97. 